\title{
Biotin deficiency and susceptibility to fatty liver and kidney syndrome in broiler chicks: reduced 6-phosphofructokinase (EC 2.7.1.11) activity but normal fructose 2,6-bisphosphate content in birds with hepatomegaly
}

\author{
By D. W. BANNISTER, D. I. SALES AND ALISON LEE \\ Agricultural and Food Research Council's Poultry Research Centre, \\ Roslin, Midlothian EH25 9PS
}

(Received 26 September 1984 - Accepted 9 May 1985)

1. In two separate experiments, using different strains, broiler chicks were reared on either a commercial-type chick mash (control) or a fatty liver and kidney syndrome (FLKS)-inducing diet.

2. In Expt $a$, chicks were killed on day 29 and in Expt b, on day 32. Body-weights and liver weights were measured, and values from those given the control ration used to construct a hepatomegaly index by employing a variant of linear discriminant analysis.

3. Application of the index to FLKS birds revealed a statistically significant bimodal distribution of liver size.

4. The birds with enlarged livers (high index) also possessed metabolic abnormalities in that 6phosphofructokinase (EC 2.7.1.11; PFK-1) activity (measured at low substrate concentration) was depressed despite the presence of normal, or even slightly elevated fructose 2,6-bisphosphate concentration.

5. This indicates the presence of an uncharacterized regulatory mechanism for PFK-1 in FLKS-susceptible birds.

The fatty liver and kidney syndrome (FLKS) of young broiler chicks is a nutritionally induced metabolic disorder in which the marginal deficiency of biotin is a key factor. The causes and metabolic changes occurring after the onset of clinical signs are understood reasonably well (for recent reviews, see Whitehead, 1978; Lindsay \& Pethick, 1983). The condition, however, remains interesting because the biochemical changes occurring before clinical signs appear are largely unknown. Greater knowledge of them should enhance our understanding of metabolic regulation in domestic fowl, particularly how it is affected by diet.

The most obvious morphological feature of birds dying, or killed while suffering from FLKS, is liver enlargement (hepatomegaly). Pearson et al. (1976) used hepatomegaly in their work by defining FLKS as present in chicks with liver weights two standard deviations greater than the mean. Subsequently Whitehead et al. (1978), feeding chicks an FLKSinducing ration, identified a proportion with enlarged livers (with no clinical signs of FLKS and possessing normal plasma glucose values) in which there were abnormally low activities of pyruvate carboxylase $(E C 6.4 .1 .1 ; \mathrm{PC})$. These birds were defined as having liver weights greater than $4 \%$ of body-weight and were shown to be at much greater risk of developing clinical FLKS than the remaining population when subjected to minor stress.

The observations described indicate the presence of metabolic abnormalities in some chicks before clinical FLKS appears. This implies that dietary and possibly other environmental factors determine the extent of an outbreak in a particular flock, rather than the degree of stress to which it is subjected. There is ample evidence favouring this view. In a series of eight field experiments performed over 2.5 years (reviewed by Whitehead \& Blair, 1976), mortality was consistently high (mean $27 \%$, range $19-43 \%$ ) when using a specific diet (Whitehead \& Blair, 1974). However, modifying the diet by increasing protein (Whitehead \& Blair, 1974) or fat content (Blair et al. 1975) reduced mortality significantly. Laboratory-scale experiments using purified diets gave similar results (Whitehead et al. 1976). 
It is clear, therefore, that a flock of chicks given an FLKS-inducing ration must contain a potentially susceptible subpopulation. Since hepatomegaly is a characteristic feature of FLKS and since liver and body-weights are easily determined, our first aim was to develop a statistical definition of hepatomegaly and see whether it could be used to identify a subpopulation within a group of birds given an FL.KS-inducing ration.

Our second aim was to determine whether the hepatomegalic subpopulation exhibited abnormal metabolic properties. For this we chose to study the regulation of 6phosphofructokinase $(E C 2.7 .1 .11$; PFK-1) and fructose-bisphosphatase $(E C$ 3 1 1.3.11; FBP-1) by the recently discovered regulatory metabolite fructose 2,6-bisphosphate (for a review of its history and properties, see Hers \& Van Schaftingen, 1982). Increased concentrations of this metabolite were found in genetically-obese mice (Claus \& Pilkis, 1982; Hue \& Van Der Werve, 1982) and rats (Hue et al. 1983) and it is believed to regulate an increased glycolytic flux in these animals associated with the high lipogenic rate. At present there is no information on this topic in FLKS-susceptible birds. Hood et al. (1976) reported increased acetyl coenzyme A carboxylase (EC 6.4 .1 .2; ACC) and Balnave et al. (1977) observed increased ATP citrate (pro-3S)-lyase (EC 4.1.3.8; ACL) activities in affected birds, both of which indicate an elevated lipogenic rate and which might be accompanied by increased fructose 2,6-bisphosphate concentration. However, the major biochemical feature of FLKS (once clinical signs of the condition have developed) is severely-impaired gluconeogenesis and, under these circumstances, reduced glycolysis would be expected to conserve the diminishing amount of glucose.

\section{MATERIALS AND METHODS \\ Birds and husbandry}

Expt a. Female broiler chicks (1-d-old) were obtained from a commercial hatchery (Ross Poultry (Great Britain) Ltd, Inverurie, Aberdeenshire) in groups of sixteen at weekly intervals. They were divided at random into two groups of eight, each of which was housed in one compartment of a heated four-tier brooder. One group received the FLKS-inducing ration of Whitehead \& Blair (1974) whilst the other (controls) was given a commercial-type chick starter diet (Bolton \& Blair, 1974). The birds were kept in the same compartment until they were 29-d-old.

Expt $b$. Experimental design was the same as for Expt a with the following modifications: (1) 1-d-old chicks were obtained from D. B. Marshall Ltd, Newbridge, Midlothian; (2) in some weeks additional groups of chicks were allocated to the FLKS-inducing diet; (3) duration was increased to $32 \mathrm{~d}$; (4) the experiment was performed at a different time of year and used a different brooder.

For both experiments water was available ad lib. and the chicks subjected to the minimum disturbance compatible with good husbandry.

\section{Experimental procedure}

On the day that each group reached the end of the experiment the chicks were selected at random (from both experimental and control groups), weighed, and killed by injection of pentobarbitone sodium (Sagatal; May and Baker Ltd, Dagenham). A portion of liver was removed as quickly as possible and frozen between aluminium tongs cooled in liquid nitrogen. This was weighed and stored at $-70^{\circ}$ for later use (see p. 537). The remaining liver was then removed, cleaned of adhering adipose tissue and weighed so that the total liver weight was determined.

This procedure was the same in both experiments and was performed between 10.00 and 12.00 hours. 


\section{Selection of livers and assay procedure}

Material collected from Expt $b$ was used. Birds were ranked according to their hepatomegaly index and divided into three groups: (1) high index, birds with an index $>2.3$; (2) low index, birds with an index $<2.3$ (the derivation of the hepatomegaly index is described in the section entitled 'statistical analysis and hepatomegaly index'); (3) controls, which received chick mash. A random sample of ten frozen livers was selected from each group for analysis.

Assays were performed in units of three, so that each unit contained one sample from each group. The order in which the samples were processed was changed from unit to unit. This procedure was chosen to minimize systematic bias in the results.

\section{Enzyme assays and fructose 2,6-bisphosphate determination}

PFK-1 was assayed at $25^{\circ}$ in the presence of $50 \mathrm{~mm}$-potassium chloride, as described by Bannister (1984), at saturating $(10 \mathrm{~mm})$ and subsaturating $(0.4 \mathrm{mM})$ concentrations of fructose-6-phosphate. FBP-1 was also determined at $25^{\circ}$ as given previously (Bannister et al. 1983), except that an additional assay at subsaturating concentration $(25 \mu \mathrm{M})$ of fructose-1-6-bisphosphate in the presence of $25 \mu \mathrm{M}$-AMP was included.

Fructose 2,6-bisphosphate was measured according to Van Schaftingen et al. (1982) as described by Bannister et al. (1984).

These assays, which were carried out in duplicate, were performed on liver samples from the same bird. Sources of chemicals were the same as in Bannister et al. (1983).

\section{Statistical analysis and hepatomegaly index}

Control birds from both experiments were checked for consistency by doing a comparison of regression of liver weight $v$. body-weight. Birds on the FLKS-inducing diet had, on average, reduced body-weights but increased liver weights, so a linear index was constructed to quantify the treatment differences. The standard method for calculating such an index is linear discriminant analysis (Fisher, 1936). As the birds on the FLKS-inducing diet were much more variable, especially for liver weight, than the controls, the usual assumption of a common covariance matrix was inappropriate. As we were concerned with departures from normal development, the discriminant function was calculated using the within-group covariance matrix from only control birds. The index was scaled so that control birds had a mean of 0 and standard deviation of 1 , and large positive values correspond to hepatomegaly.

The index values of birds given the FLKS-inducing diet were tested for bimodality by finding the partition which maximized the ratio, between-birds:within-birds sums of squares. The significance of the observed ratio was assessed by simulation. This test is sensitive to skewness as well as bimodality, so the kernel density estimate test described by Silverman (1981) was also applied.

All these analyses were carried out on both the original weights and on their logarithms; results for the latter are to base 10 throughout.

\section{RESULTS}

Graphs of liver weight plotted $v$. body-weight are shown in Fig. 1 for controls and Fig. 2 for experimental birds. These suggest that experimental birds were more variable than controls and that the variance of liver weight increased with body-weight. The latter suggests that analysis based on the logarithm of the weights would be the method of choice. Both analyses were carried out and gave very similar results; however, the log analysis was slightly more sensitive. 


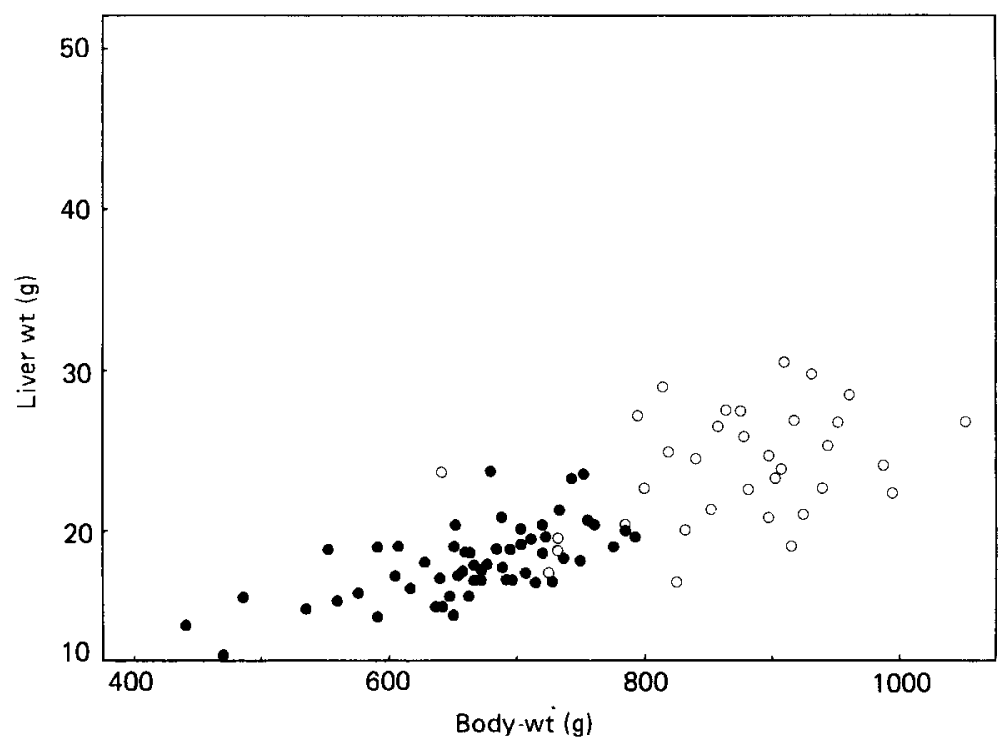

Fig. 1. Relation between liver weight and body-weight for two strains of broiler chick given a commercial-type ration. Values are for two experiments using (Expt a) thirty-four female Ross broiler chicks $(\mathrm{O})$ and (Expt b) fifty-six female Marshall chicks ( ). Expt a was terminated on day 29 and Expt b on day 32. Husbandry conditions are given on p. 536 and feed (Bolton \& Blair, 1974) was available at all times.

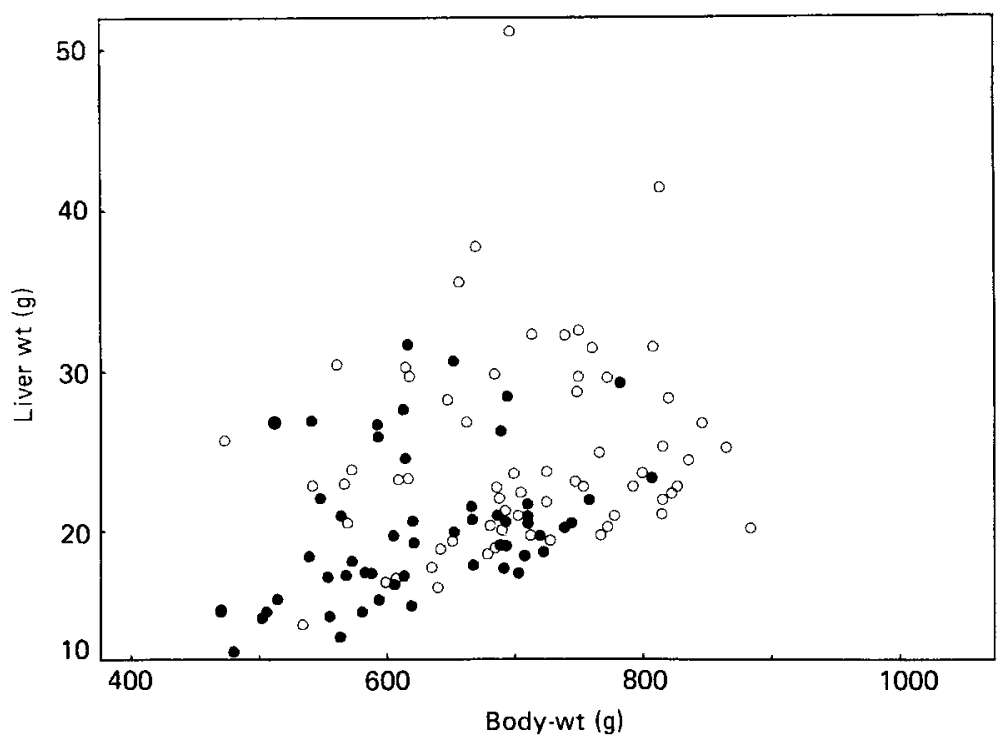

Fig. 2. Relation between liver weight and body-weight for two strains of broiler chick given a fatty liver and kidney syndrome-inducing ration. Values are for two experiments using (Expt a) fifty-four female Ross broiler chicks $(O)$ and (Expt b) sixty-six female Marshall chicks $(\mathbf{O})$. Expt a was terminated on day 29 and Expt b on day 32. Husbandry conditions are given on p. 536 and feed (Whitehead \& Blair, 1974) was available at all times. 
Table 1. Estimated weights of indices used to construct the hepatomegaly index

(The hepatomegaly index depends on the relation $(a+b L-B) / c$, where $L$ is liver weight and $B$ is bodyweight, or their logarithms. Values for liver and body-weights were collected from two separate experiments using female Ross or Marshall broiler chicks. Full experimental details are given on pp. 536-537.)

\begin{tabular}{lccc}
\hline \hline & \multicolumn{3}{c}{ Index wt } \\
\cline { 2 - 4 } & $a$ & $b$ & $c$ \\
\hline Linear & 93 & 32 & $85 \cdot 3$ \\
Log-linear & 1.7 & 0.9 & 0.049 \\
\hline \hline
\end{tabular}

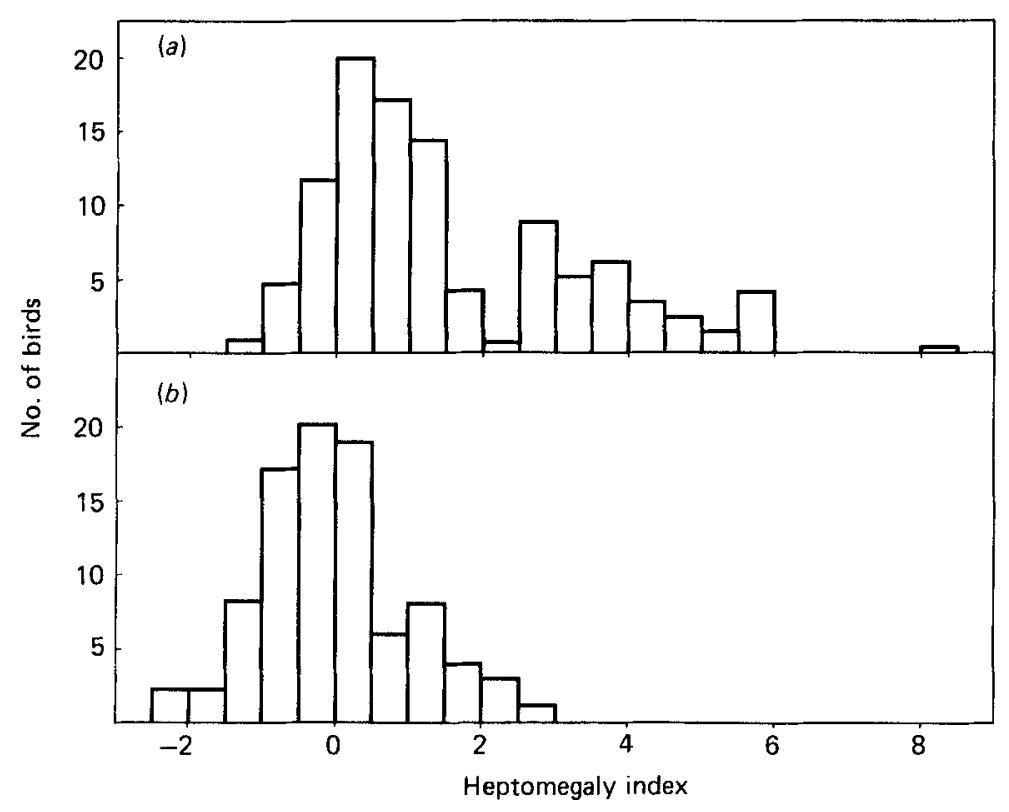

Fig. 3. Frequency distribution of hepatomegaly index in broiler chicks given either a fatty liver and kidney syndrome (FLKS)-inducing ration or a commercial-type ration. The log-linear hepatomegaly index (for details, see p. 537) was applied to chicks from two independent experiments. (a) Values for the FLKS ration are taken from Fig. 2, (b) values for controls, see Fig. 1.

Within the two experiments there was no evidence of differences between the weekly groups of chicks in either liver or body-weight. The birds from the second experiment were larger, as expected from the $3 \mathrm{~d}$ extra duration. However, the regression of liver weight $v$. body-weight did not differ significantly between the two experiments, so the construction of the index was based on the pooled values. The estimated linear and $\log$-linear indices are given in Table 1. Histograms of the corresponding index values for the log-linear index are plotted in Fig. 3. The apparent bimodality of the histograms for the birds on the FLKS-inducing diet is highly significant $(P<0.001)$ according to the sums of squares test, with the optimal split occurring between index values 2.05 and 2.51 . The kernel density estimate test gave three separate modes $(P<0.05)$, separating off a single observation at index value 8.04 as an outlier from the two main groups.

If we use this index to classify the birds on the experimental diet, then thirteen of fifty-four $(24 \%)$ Ross birds and twenty-five of sixty-six ( $38 \%$ ) Marshall birds were considered to show 
Table 2. Body-weights and liver weights of two strains of broiler chick reared on a fatty liver and kidney syndrome (FLKS)-inducing diet

(Mean values with their standard deviations. In Expt a, Ross chicks were used and they were killed on day 29. For Expt b, Marshall chicks were used and killed on day 32. The heptomegaly index was constructed as described on p. 537 using liver weight and body-weight values collected at the same time from birds reared on a control ration. High-index birds are those with a value of 2.3 or greater)

\begin{tabular}{ccccccc}
\hline \hline & & \multicolumn{2}{c}{ Body-wt (g) } & & \multicolumn{2}{c}{ Liver wt (g) } \\
\cline { 5 - 6 } & No. of birds & Mean & SD & & Mean & SD \\
\hline Expt a & & & & & \\
FLKS diet: & & & & & \\
High index & 13 & 616 & 74 & & $26 \cdot 8$ & $3 \cdot 1$ \\
Low index & 41 & 634 & 82 & & $18 \cdot 2$ & $2 \cdot 6$ \\
All & 54 & 630 & 80 & $20 \cdot 3$ & $4 \cdot 6$ \\
Control diet & 56 & 666 & 75 & $18 \cdot 1$ & $2 \cdot 3$ \\
Expt b & & & & & \\
FLKS diet: & & & & & \\
High index & 25 & 665 & 90 & $30 \cdot 1$ & $6 \cdot 7$ \\
Low index & 41 & 732 & 80 & $21 \cdot 3$ & $2 \cdot 9$ \\
All & 66 & 706 & 89 & $24 \cdot 7$ & $6 \cdot 3$ \\
Control diet & 34 & 870 & 86 & $23 \cdot 9$ & $3 \cdot 6$ \\
\hline \hline
\end{tabular}

hepatomegaly; these frequencies did not differ significantly. A summary of means and standard deviations of liver and body-weight for these groups is given in Table 2. The values show that the FLKS-inducing ration reduced body-weight by approximately $6 \%$ in Expt a and $19 \%$ in Expt $b$ when compared with chick mash. The reduction was greater in high-index birds and less in low-index birds.

Mortality among birds fed on chick mash was zero for Expt a and $1(2.9 \%)$ for Expt b. The corresponding values for the FLKS-inducing diet were $2(3.5 \%)$ and $11(14.3 \%)$.

Means for fructose 2,6-bisphosphate concentration and enzyme activities are given in Table 3. Although the concentration of the metabolite was higher in high-index birds compared with low-index birds, there were no significant differences between the three groups. Maximum initial reaction velocity $\left(V_{\max }\right)$ for both PFK-1 and FBP-1 were also similar in all three groups. However, activities determined at low substrate concentrations (initial reaction velocity $v / V_{\max }$ ) were depressed. This was unexpected for PFK-1 because fructose 2,6-bisphosphate is known to be a potent activator of the enzyme (Van Schaftingen et al. 1980), but is in keeping with the expectation for FBP-1 which it inhibits (Van Schaftingen \& Hers, 1981).

By chance, the bird with an index value of 8.04 was one of the ten sampled from the high-index group. There was some indication that it had a lower $V_{\max }$ for FBP-1 $(P<0.05)$; its removal would raise the mean from 700 to $744 \mu \mathrm{mol} / \mathrm{g}$ liver per $\mathrm{h}$. However, there was no evidence that this bird differed for any other biochemical trait.

For each trait there was no difference between controls and low-index birds. There was also no evidence of an association of enzyme activity and hepatomegaly index within the three groups.

\section{DISCUSSION}

\section{Hepatomegaly}

Pearson et al. (1976) and Whitehead et al. (1978) showed hepatomegaly to be a prominent feature of FLKS. However, the definitions they used are arbitrary and it is our intention, by using a statistical approach, to provide a more satisfactory basis for defining liver enlargement in this condition. 
Table 3. Fructose 2,6-bisphosphate concentration, 6-phosphofructokinase (EC 2.7.1.11; $P F K-1)$ and fructose-bisphosphatase (EC 3.1.3.11; FBP-1) activities in livers of birds given either a fatty liver and kidney syndrome (FLKS)-inducing diet or chick mash

(Ten frozen liver samples from Expt $b$ were allocated to each of three groups as follows: (1) high index, hepatomegaly index greater than $2 \cdot 3 ;$ (2) low index, hepatomegaly index less than $2 \cdot 3 ;$ (3) chick mash. They were assayed by the methods given on p. 537. For PFK-1 $V_{\max }$ was measured at $10 \mathrm{~mm}$-fructose6-phosphate and $v$ at $0.4 \mathrm{mM}$. For FBP-1, $V_{\max }$ was determined at $250 \mu \mathrm{M}$-fructose-1,6-bisphosphate and $\nu$ at $25 \mu \mathrm{M}$ in the presence of $25 \mu \mathrm{M}-\mathrm{AMP}$ )

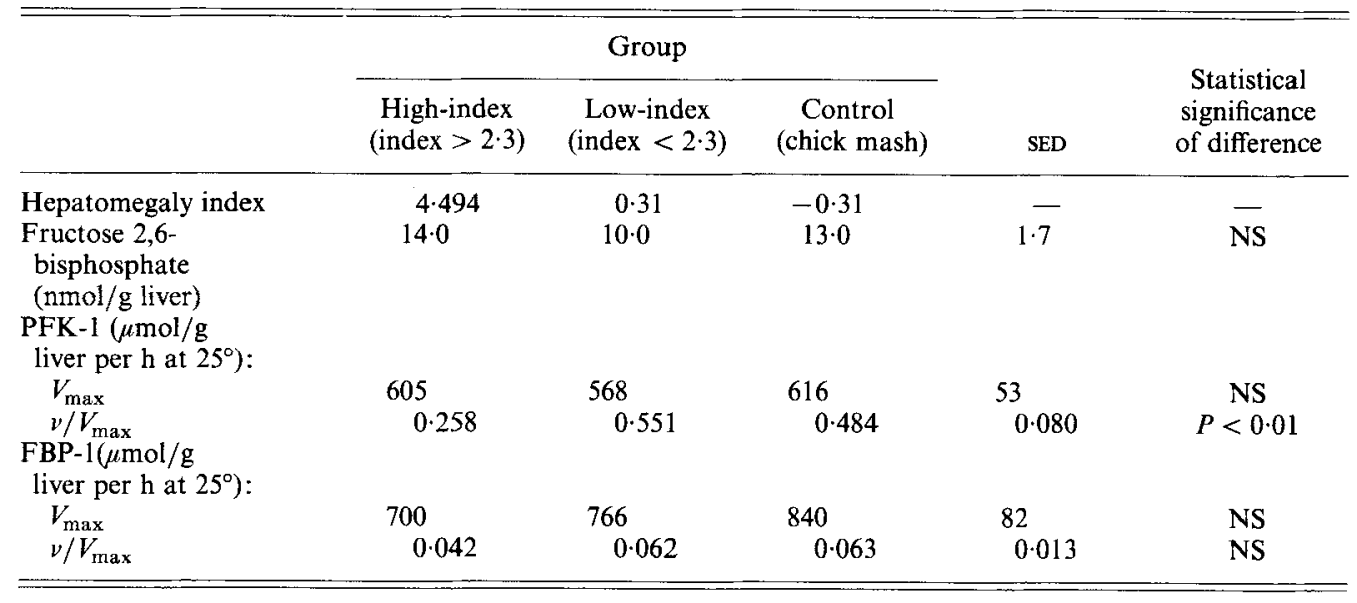

NS, not significant; SED, standard error of difference between means; $v$, initial reaction velocity; $V_{\max }$, maximum initial reaction velocity.

By using the index, birds given the FLKS-inducing ration separated into two, or possibly three, populations (Fig. 3). This is a significant finding because it implies that FLKSsusceptible birds have diverged from the normal path of development. Further support for the idea of divergence comes from the greater differences in weight, and perhaps also mortality, observed in Expt b, when the birds were kept 3 d longer.

A satisfactory feature of the hepatomegalic subpopulation was that its size is compatible with existing knowledge about FLKS mortality. Approximately $31 \%$ of chicks (for both experiments) fell into this category; the value rises to $38 \%$ if we assume that all premature mortality was due to FLKS (no post-mortem examinations were performed). These values are at the upper end of the range $19-43 \%$ mortality found in a series of field experiments using the same dietary formulation as employed here (see Whitehead \& Blair, 1976). In practice, the potentially-susceptible population is expected to be greater than mortality values collected in field work because some birds suffering FLKS subsequently recover (Whitehead, 1975). Furthermore, it is not certain that all such birds in a sizeable population will encounter stress of sufficient severity to precipitate clinical signs of the syndrome.

The biochemical evidence also supports the existence of a separate, high-risk population in that the observed differences occurred only in high-index birds, there being no differences between the low-index and control groups.

\section{Regulation of enzyme activity}

Fructose 2,6-bisphosphate activates PFK-1 and inhibits FBP-1 when measured at low substrate concentrations (see review by Hers \& Van Schaftingen, 1982) and, hence, is a key intracellular regulator of the balance between glycolysis and gluconeogenesis. Increased concentrations have been found under conditions, such as genetically-determined obesity, in which elevated glycolytic flux occurs (Claus \& Pilkis, 1982; Hue \& Van Der Werve, 1982; 
Hue et al. 1983). We were, therefore, interested to examine the situation in birds at risk of developing FLKS.

The results are clearcut but their interpretation is not. Hood et al. (1976) found increased ACC activity in birds suffering FLKS and proposed that elevated lipogenesis is a significant metabolic feature of the syndrome. Balnave et al. (1977) supported the idea by finding increased ACL activity and, more recently, Bannister et al. (1983) found increases in a number of lipogenic enzymes, although the experimental design was different from that of the earlier work. Thus there is evidence for increased lipogenic capacity both before and after the onset of clinical signs. The fact that fructose 2,6-bisphosphate concentration was slightly greater in high-index birds than in low-index ones (although there was no overall difference between the three groups, see Table 3 ) is entirely consistent. So also is the modest reduction in FBP-1 $v / V_{\max }$ value. However, the finding that PFK-1 had a lower $v / V_{\max }$ value in high-index birds, rather than remaining normal or even slightly increased, was not expected and is currently unexplained.

Considering first the physiological reason for inhibition we suggest that, since FLKSsusceptible birds have very low PC activity (Whitehead et al. 1978) and gluconeogenesis is presumably severely impaired, lowered PFK-1 activity represents a means of conserving the limited supply of glucose. Second, it is important to understand the mechanism causing the low $v / V_{\max }$ value because it is clearly not due to lowered fructose 2,6-bisphosphate concentration. There are at least three possibilities. (a) Increased concentration of a high-molecular-weight inhibitor of PFK-1 in high-index birds. In this context it is worth noting that a protein inhibitor has recently been reported in rat intestinal mucosa (Kellet \& Robertson, 1984). (b) There is a reduced concentration of the high-molecular-weight regulatory factors described by Kruep \& Dunaway $(1984 a, b)$ in high-index birds. (c) PFK-1 in high-index birds is more extensively phosphorylated because this form of the enzyme is more susceptible to inhibition by ATP (Kitajima et al. 1983).

\section{Concluding remarks}

Our evidence showed that by the time birds given an FLKS-inducing diet reached the age when they were susceptible to clinical FLKS (about 4 weeks), they had separated into two distinct subpopulations identifiable by using statistical techniques. In one there was a similar relation between liver weight and body-weight to that found in the control population. Furthermore, it did not differ in the metabolic indices studied here. The other subpopulation had enlarged livers and reduced body size and was similar in size to that succumbing to FLKS in earlier field trials (Whitehead \& Blair, 1976). The subpopulation was also metabolically distinct in having a reduced PFK-1 $v / V_{\max }$ value, although the reason for the lowered activity and the mechanism causing it are uncertain. Thus the metabolic results amply confirm the statistical separation into at least two subpopulations.

The authors are grateful to Mr James MacKinlay for his technical assistance.

\section{REFERENCES}

Balnave, D., Cumming, R. B. \& Sutherland, T. M. (1977). British Journal of Nutrition 38, 319-328.

Bannister, D. W. (1984). International Journal Biochemistry 16, 895-899.

Bannister, D. W., Lee, A., Whitehead, C. C. \& Griffin, H. D. (1984). International Journal of Biochemistry 16, 1301-1305.

Bannister, D. W., O'Neill, I. E. \& Whitehead, C. C. (1983). British Journal of Nutrition 50, $291-302$.

Blair, R., Whitehead, C. C. \& Teague, P. W. (1975). Research in Veterinary Science 18, 76-81.

Bolton, W. \& Blair, R. (1974). Poultry Nutrition, Bulletin no. 174, p. 128. London: H.M. Stationery Office.

Claus, T. H. \& Pilkis, S. J. (1982). Biochemical and Biophysical Research Communications 109, 664-668.

Fisher, R. A. (1936). Annals of Ergenics 7, 179-188. 
Hers, H.-G. \& Van Schaftingen, E. (1982). Biochemical Journal 206, 1-12.

Hood, R. L., Johnson, A. R., Fogarty, A. C. \& Pearson. J. A. (1976). Australian Journal of Biological Science 29, $429-441$.

Hue, L. \& Van Der Werve, G. (1982). FEBS Letters 145, 263-266.

Hue, L., Van Der Werve, G. \& Jeanrenaud, B. (1983). Biochemical Journal 214, 1019-1022.

Kellet, G. L. \& Robertson, J. P. (1984). Biochemical Journal 220, 601-604.

Kitajima, S., Sakakibara, R. \& Uyeda, K. (1983). Journal of Biological Chemistry 258, 13292-13298.

Kruep, D. A. \& Dunaway, G. A. (1984a). Archives of Biochemistry and Biophysics 235, 504-511.

Kruep, D. A. \& Dunaway, G. A. (1984b). Archives of Biochemistry and Biophysics 235, 512-520.

Lindsay, D. W. \& Pethick, D. W. (1983). In Dynamic Biochemistry of Animal Production: World Animal Science, vol. A3, pp. 431-480 [P. M. Riis, editor]. Amsterdam: Elsevier.

Pearson, J., Johnson, A. R., Hood, R. L. \& Fogerty, A. C. (1976). Australian Journal of Biological Science 29, 419-428.

Silverman, B. W. (1981). Journal of the Royal Statistical Society, Series B 43, 97-99.

Van Schaftingen, E. \& Hers, H.-G. (1981). Proceedings of the National Academy of Sciences, USA 78, 2861-2863.

Van Schaftingen, E., Hue, L. \& Hers, H.-G. (1980). Biochemical Journal 192, 897--901.

Van Schaftingen, E., Ledener, B., Bartrons, R. \& Hers, H.-G. (1982). European Journal of Biochemistry 129, 191-195.

Whitehead, C. C. (1975). Research in Veterinary Science 18, 32-35.

Whitehead, C. C. (1978). In Handbook Series in Nutrition and Food, Sect. E, vol. 2, pp. 65-93 [M. Rechagle, editor]. Florida: CRC Press Inc.

Whitehead, C. C., Bannister, D. W. \& Cleland, M. E. (1978). British Journal of Nutrition 40, 221-234.

Whitehead, C. C., Bannister, D. W., Evans, A. J., Siller, W. G. \& Wright, P. A. L. (1976). British Journal of Nutrition 35, 115-125.

Whitehead, C. C. \& Blair, R. (1974), Research in Veterinary Science 17, 86-90.

Whitehead, C. C. \& Blair, R. (1976). Research in Veterinary Science 21, 141-145. 